\title{
Degassing of FC-72 in Microgravity
}

\author{
Danielle Weiland ${ }^{1}$, Kevin Crosby $^{1}$, Nancy Hall ${ }^{2}$ \\ ${ }^{1}$ Carthage College, Kenosha, WI, USA \\ ${ }^{2}$ NASA Glenn Research Center, Cleveland, Ohio, USA
}

\begin{abstract}
The Carthage College Microgravity Team designed and built a system to degas FC-72 during microgravity conditions through the NASA SEED program. FC-72 is an electronic coolant with low boiling point and surface tension that would be an ideal coolant for use in a two-phase cooling system onboard spacecraft [3M, 2013]. The goal of the project was to remove the dissolved gases from liquid FC72 to improve the efficiency of flow boiling in microgravity as part of the Flow Boiling and Condensation Experiment conducted jointly by NASA Glenn Research Center (GRC) and Purdue University. A radial membrane contactor was used to remove the gases from the liquid during microgravity. Data from the lab was compared with the parabolic flight data to determine the relative effectiveness of the radial membrane contactor in reduced gravity.
\end{abstract}

\section{Introduction}

In the work reported here, we demonstrate a method of de-gassing of the dielectric coolant FC72 (perfluorohexane) using a radial membrane contactor (RMC). The method was tested in the reduced gravity environment of parabolic flight. The tests were conducted as part of NASA's Systems Engineering Education Discovery (SEED) program. A team of seven undergraduate students designed and built a flow-loop to measure and characterize the efficiency of the RMC in extracting dissolved oxygen from the liquid FC-72. Dissolved oxygen content as a function of flow rate and ambient conditions were measured and compared with 1-g laboratory testing. Data from the microgravity tests will be used to inform the design of the Flow Boiling and Condensation Experiment (FBCE) being developed at NASA-GRC [3M, 2013].

The FBCE scheduled for deployment on the International Space Station (ISS) in 2017 will provide the first data on heat transfer rates and condensation dynamics in reduced gravity. The FBCE will serve as a first step toward developing two-phase cooling systems for future spacecraft. A significant technical challenge of two phase heat exchange systems is the removal of dissolved gasses introduced into the coolant fluid during condensation. Dissolved gasses

The authors gratefully acknowledge the Wisconsin Space Grant Consortium for financial support and the Reduced Gravity Office at NASA Johnson Space Center for support of the Systems Engineering Educational Discovery (SEED) program. The authors also acknowledge the useful contributions made by the Carthage Microgravity Team student members who worked on this project include Daisy Bower, Amelia Gear, Eli Favela, Kevin Lubick, Steven Mathe, John Robinson, and Seth Schofield. 
(primarily atmospheric oxygen) reduce the effective heat capacity of the fluid, making the cooling less efficient. Therefore, a method of degassing coolant in microgravity environments is needed before two-phase cooling systems can be practically implemented.

\section{Background}

The purpose of the current experiment is to test a method of degassing a next-generation coolant using a radial membrane contactor. We measure the rate of atmospheric oxygen removal in a saturated liquid coolant and compare these measurements to those obtained in ground testing. One candidate fluid for a two-phase cooling system aboard the ISS is a member of the perfluorohexane family. The commercial product FC-72 (manufactured by 3M) has been chosen as the perfluorohexane of choice for this experiment due to low boiling point, the fact that it is a liquid at STP, its high dielectric constant, as well as the absence of toxic effects in humans. In this experiment we test the ability of a Radial Membrane Contactor (RMC) to remove dissolved gas from FC-72 in a microgravity environment [3M, 2013] using parabolic flights provided by NASA.

The RMC exploits the principles of capillary action to remove dissolved gas from a liquid without removing or chemically modifying the liquid [Liqui-cel, 2013]. Unlike degassing techniques used in industry, which typically involve cycles of boiling the fluid followed by vacuum exposure, the present technique does not depend on gravity to function properly, making it an ideal candidate for working aboard the ISS. The RMC has three main parts - a vacuum port, a series of thin, radially-aligned glass tubes wrapped around the flow tubing and a thin membrane that acts as an interface between the liquid and the induced vacuum. This setup allows a small vacuum pump to pull dissolved gas out of the fluid flowing through the RMC. Figure 1 shows a diagram of the RMC.

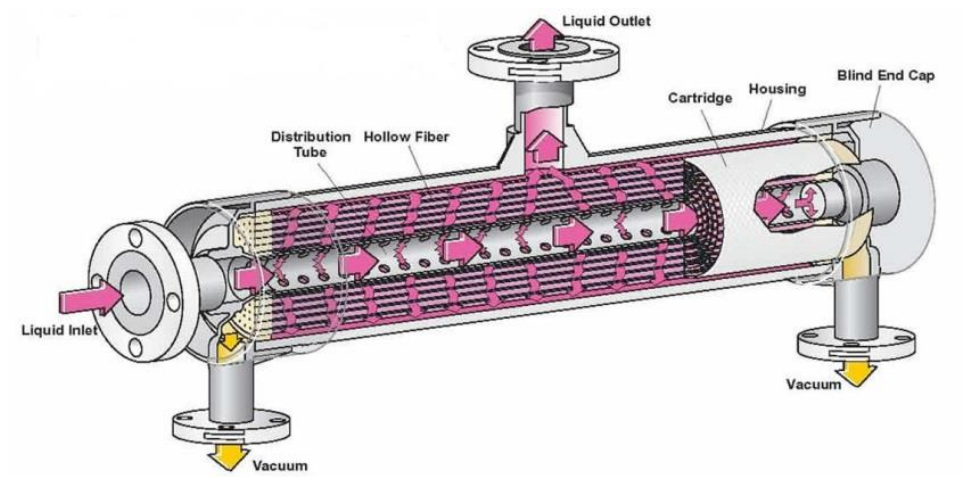

Figure 1: Diagram of the Radial Membrane Contactor [3M, 2013]. 


\section{Properties of FC-72}

Perflourohexane (FC-72) is a clear, colorless, odorless liquid with no known hazards. Relevant properties of FC-72 are listed in Table 1[3M, 2013].

\begin{tabular}{|l|c|}
\hline Flammable Limit: & Nonflammable \\
\hline Boiling Point: & $50-60 \mathrm{C}$ \\
\hline Pour Point: & $-90 \mathrm{C}$ \\
\hline Density: & $1.7 \mathrm{~g} / \mathrm{ml}$ \\
\hline Vapor Density: & $\sim 11.7($ at $20 \mathrm{C})$ \\
\hline Vapor Pressure: & $\sim 1.7$ \\
\hline Specific Gravity: & $>1$ \\
\hline Evaporation Rate: & $\sim 232 \mathrm{mmHg}($ at $20 \mathrm{C})$ \\
\hline Viscosity: & $\sim 0.42$ centistoke $($ at $20 \mathrm{C})$ \\
\hline
\end{tabular}

Table 1: Properties of FC-72

There are a few key properties that make FC-72 ideal for use in heat exchange systems. One such property is its low viscosity. Low viscosity fluids require comparably smaller pump pressure heads and therefore require less energy to circulate. Another property that makes FC-72 favorable for use in the present application is its high dielectric constant which will prevent any damage to electronics during a spill or leak. Also, there are no known hazards to humans in contrast to ammonia which is commonly used in two fluid, single phase cooling systems and is extremely toxic.

\section{Heat Exchange Systems}

Dissipation of environmental heat is a problem in large spacecraft. Under normal gravitational conditions, heat diffuses through the internal atmosphere of the spacecraft by convection until it can be dissipated externally. In the absence of gravity, natural convection does not occur and alternate methods of heat transfer must be used.

The current method of environmental cooling on the International Space Station is a two-fluid, single-phase system in which liquid water is circulated through Station to absorb heat, and then is brought into conductive contact with pipes containing anhydrous ammonia that dissipate this heat into space. The two-fluid, single-phase system on the ISS relies on sensible heat transfer (no phase change) and is less efficient than two-phase, single fluid heat transfer systems relying on latent heat exchange. Long-duration spaceflight will necessitate advancements in spacecraft heat dissipation and will likely rely on two-phase heat exchange systems. 
In general, the use of a single-phase coolant loop, such as the one currently on the ISS, poses several problems that result in lowered cooling efficiencies. The fluid in contact with the external space environment (the ammonia) must have its contact time monitored and limited to prevent it from freezing. Finally, the use of ammonia poses health risks to crewmembers in the event of leaks.

An alternative, more efficient, approach is to use a liquid with a much lower boiling temperature in a two-phase cooling system. In two-phase systems, coolant has a low enough boiling temperature that when it absorbs heat from the station it will begin to boil. The vapors are then cycled outside the station, where they condense back into a liquid that can be cycled back into the station for reuse. The two phase system is, in general, more efficient than a single phase cooling system. However, during the boiling and condensation processes gas dissolves into the liquid. This process reduces the heat capacity and latent heat of the fluid, making the cooling less efficient. Therefore, a method of degassing coolant in microgravity environments is needed before two-phase cooling systems can be practically implemented.

\section{Research Objectives}

The central objective of the present study is to demonstrate the effectiveness of using a radial membrane contactor to degas a fluid in a zero-g environment. Radial membrane contactors have been used for many years in small laboratory experiments to remove dissolved gases from fluids. Extensive ground testing was performed to test the effectiveness of degassing both water and FC-72 in normal gravity conditions.

Another research objective is to demonstrate the radial membrane contactor's ability to bring fully oxygenated FC-72 down to its vapor pressure. If this is accomplished we know there are no dissolved gases present in the liquid and the efficiency of the FC-72 for cooling in microgravity would be maximized.

The zero-g data was obtained through participation in NASA's Systems Engineering Exploration Discovery (SEED) student microgravity parabolic flight program. We used the ground data as a reference to determine the effectiveness of the radial membrane contactor in microgravity conditions. The data collected on flight should closely represent the data from in lab testing.

\section{Experimental Design}

The experiment has multiple subsystems including the structural, electrical and hydraulic subsystems. The structural subsystem is comprised of all parts of the rig that house the electrical and hydraulic subsystems for safety. Figure 2 is a SolidWorks design showing the structural frame of the rig. 


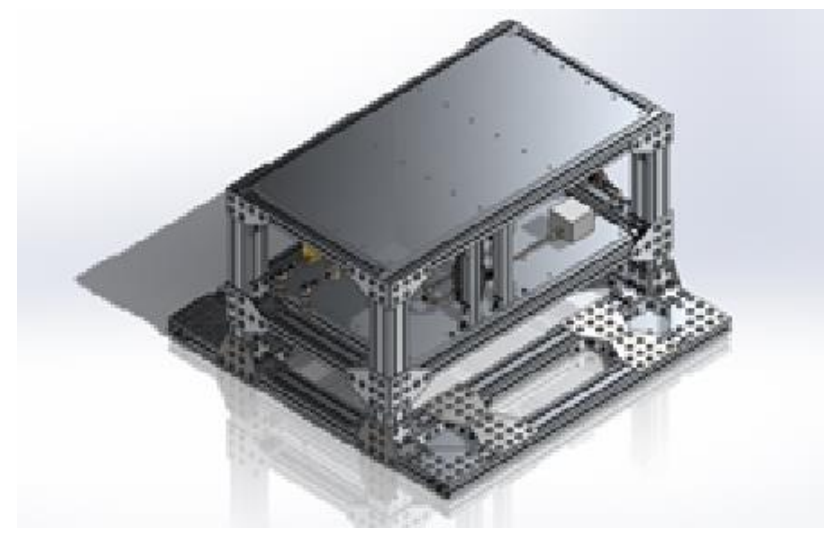

Figure 2: Structural Frame of Experiment (28.4in x 28.0in x 15.5in);

The experimental flow loop sits horizontally on the middle deck of the rig.

The structural frame of this rig is constructed from 6360 T6 aluminum extrusions, manufactured by TSLOTS, Inc. [TSLOTS, 2013]. These extrusions feature a slotted profile. These extrusions are fastened to each other using joint pieces machined from 6063 aluminum with zinc-plated A36 steel nuts and bolts. The extrusions have a spring locking feature, which creates a vibrationproof connection when tightened to the proper torque.

The electrical subsystem is comprised of all components that require power to run including the software used for data saving and analysis. The electrical subsystem is shown in Fig. 3. The design requires four primary sensors to record dynamic properties of the flow loop. These sensors measure temperature, pressure, flow rate and the concentration of dissolved $\mathrm{O} 2$ and are sampled by the analog to digital converter (ADC) at a rate of $1 \mathrm{~Hz}$. Additionally, the electrical subsystem includes programmable relays which control solenoid valves. The valves in turn allow the software to route the fluid through the membrane filter during microgravity periods on the parabolic flights.

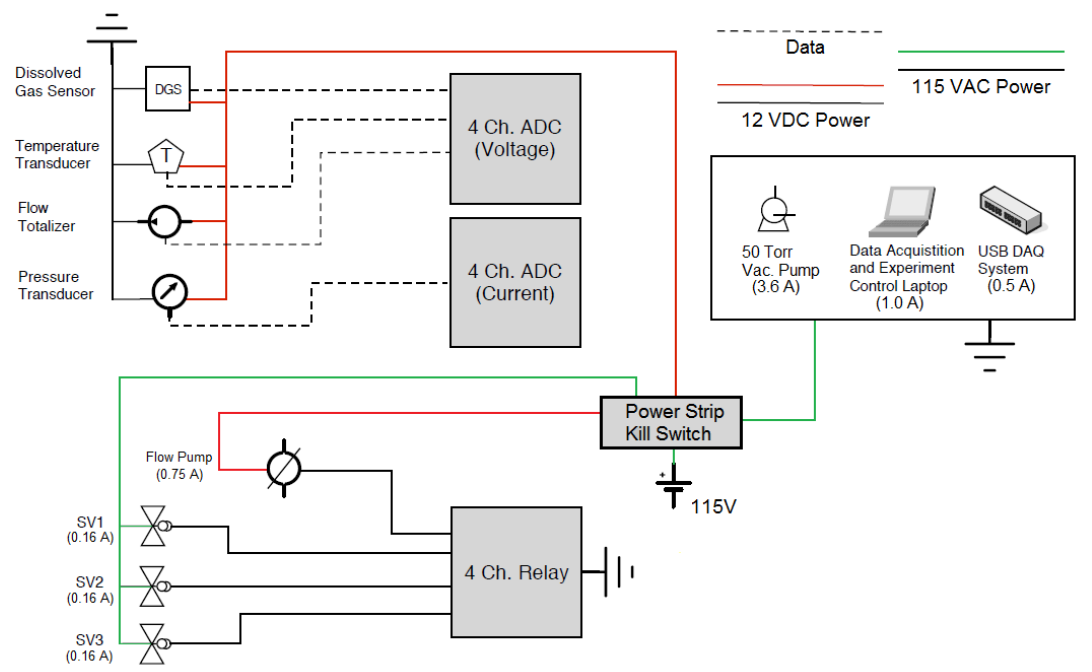

Figure 3: Electrical Subsystem 
This experiment uses a tablet laptop equipped with a touch screen interface to allow for easy use in the microgravity environment of the aircraft. The laptop runs National Instruments LabVIEW software to control the data acquisition and fluid flow. A National Instruments four-channel USB chassis, item \# NI-9174, is used to connect the relay module and DAQ modules to the computer via a USB cable [National Instruments, 2013].

The remaining subsystem constitutes the bulk of the experiment: the hydraulic subsystem. This includes all components that hold or transfer fluid. To test the effectiveness of the RMC at removing gas from FC-72 in microgravity conditions, the RMC is inserted into a flow loop designed to pump FC-72 through the RMC solely during the microgravity portions of flight. This flow loop is illustrated in Figure 4.

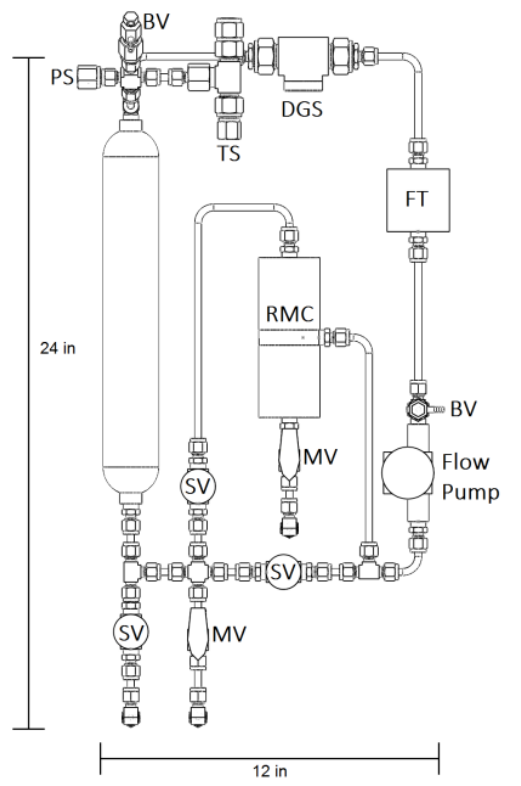

Figure 4: Flow loop with labeled components. SV-Solenoid Valve; MV-Manual Valve; BV-Bleed Valve; FT-Flow Totalizer; DGS - Dissolved Gas Sensor; TS - Temperature Sensor; PS - Pressure Sensor

The flow loop consists of the following components: the RMC, Flow Pump, Flow Totalizer, dissolved gas sensor, temperature probe, pressure transducer, a 500ml fluid reservoir, and assorted manual valves and solenoid valves to help control fluid flow. The FC-72 flows through all of the above components inside $1 / 4$ inch stainless steel tubing. Dissolved gasses in the FC-72 are extracted through the capillary tubes inside the RMC and removed via an oil-less vacuum pump. During flight, we have roughly $400 \mathrm{ml}$ of FC-72 in the flow loop. 
Signals from the dissolved gas sensor, the temperature probe and the pressure transducer are acquired by a USB-based Data Acquisition (DAQ) module for data collection by a laptop. Additionally, the solenoid valves and the flow pump are controlled by relays driven by the DAQ module so that the laptop software can control the flow of the fluid through the flow loop [National Instruments, 2013].

During the first flight, the FC-72 is cycled through the RMC only during microgravity portions of the flight, and routed around the RMC during hypergravity. The FC-72 is cycled through the flow loop for the first 14 parabolas, and after data collection, will be bubbled for the $2 \mathrm{~g}$ portion of parabola 15 , and the entirety of parabola 16 . The bubbling process is necessary to reintroduce oxygen into the FC-72. The fluid will then equilibrate for one parabola, circulating in a mode that bypasses the RMC, before the process begins again. During the second flight, the FC-72 is not reintroduced to oxygen and is degassed during all 30 zero-gravity portions of flight.

During the hypergravity portions of the flight, degassing of FC-72 ceases and the pressure and temperature of the gas in the flow loop are measured to obtain the dissolved gas content using the volume measurement obtained from the flow totalizer and ideal gas laws. Additionally, an in-line Dissolved Gas Sensor provides a secondary measurement of the partial pressure of dissolved gas in the FC-72.

\section{Results}

Figure 5 is a graph of the lab data when FC-72 was degassed for 35 minutes. The black line on the graph shows the measured pressure of the flow loop during the de-gassing and the red line is the percent of dissolved oxygen remaining in the FC-72. The blue dashed line is the vapor pressure of FC-72 - $26.9 \mathrm{kPa}$ at STP.

The flow loop began at room temperature with $500 \mathrm{ml}$ of FC-72 in it and was cycled with a flow rate of $480 \mathrm{ml} / \mathrm{min}$.

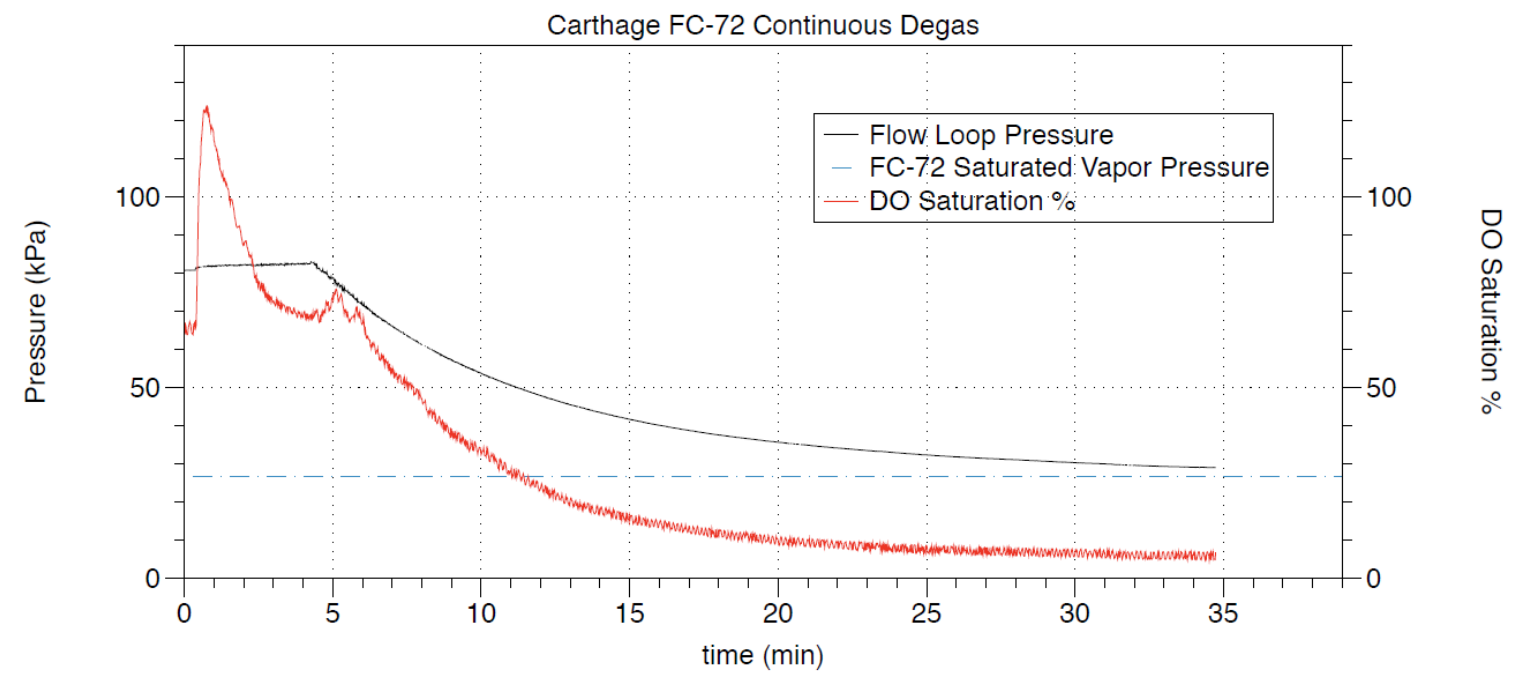

Figure 5: FC-72 Continuous Degas of FC-72 in 1-g (lab) conditions. Shown here are both pressure in the flow loop and Dissolved Oxygen (DO) saturation. 
Figure 6 shows the pressure drop of the system during ground testing as well as comparison data for the first flight day. The flight data shows data retrieved during zero-gravity portions of the flight to compare with the continuous degas lab data.

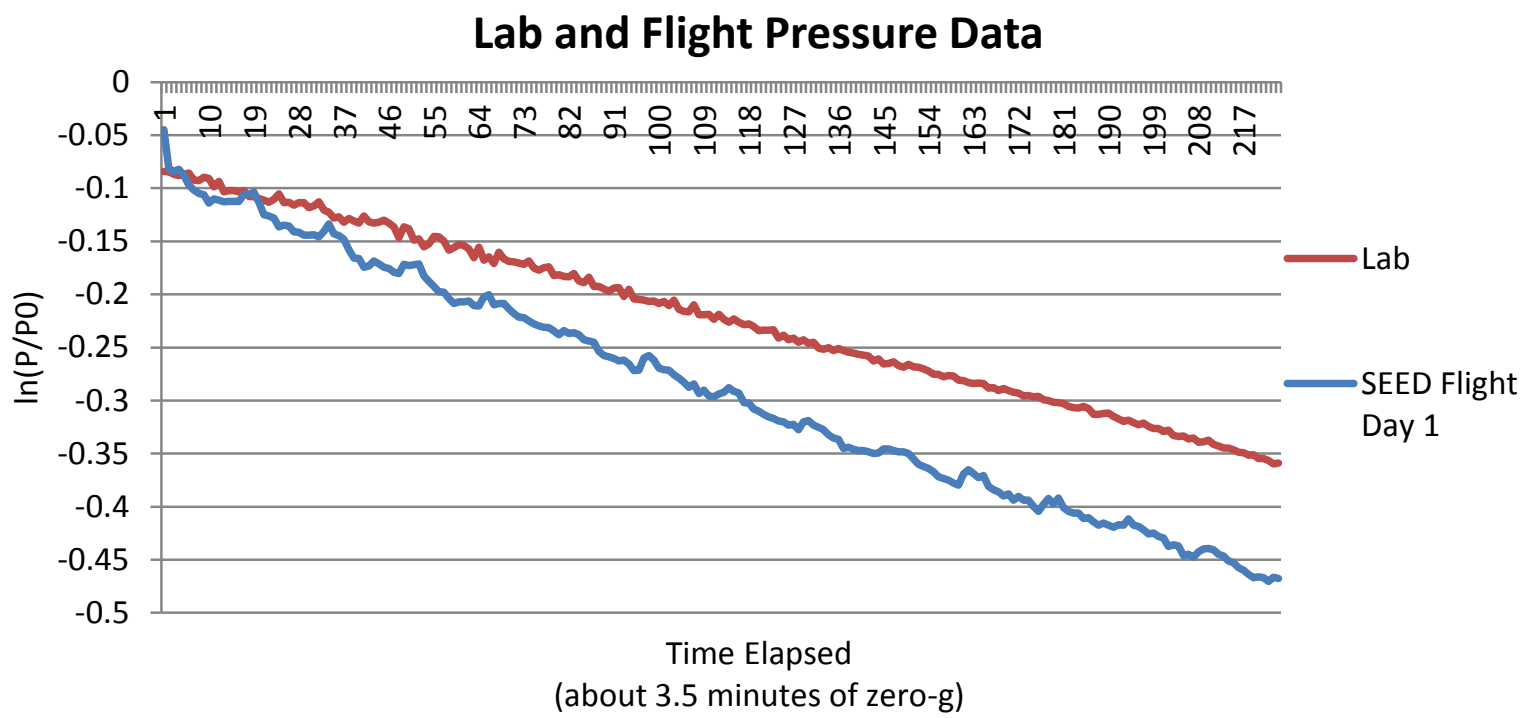

Figure 6: Lab and Flight Pressure Data for continuous degas (lab) and 0-g only (flight).

\section{Discussion}

The central objective of the study reported here was to determine the effectiveness of using a radial membrane contactor to degas a fluid in a zero-g environment with the results to be used to supplement the FBCE.

One goal of the study was the effort to degas the FC-72 down to its vapor pressure of $26.9 \mathrm{kPa}$. During the flight time available, the lowest pressure of the FC-72 achievable was $52 \mathrm{kPa}$. In $1-\mathrm{g}$ lab testing the lowest pressure obtainable was $29.2 \mathrm{kPa}$ and the amount of dissolved oxygen remaining was $5 \%$.

It was shown that the RMC works in the lab but was incapable of bringing the FC-72 to its vapor pressure indicating that we were not able to remove all dissolved gases from the liquid. During the 35 minute in-lab degassing, data from our flow totalizer showed the flow rate decreasing. The reason for this may be that boiling of the FC-72 was occurring. The flow pump and totalizer only work with liquids and therefore would stop working with only gas in the system. 
If we continued to try and degas the remaining liquid further, the pressure would begin to rise again instead of drop. A potential solution to this would be the use of more FC-72 in the system to slow the onset of boiling.

A possibility for why the degassing rate was lower in zero-g than in 1-g is that flow boiling onset occurs at higher gas pressure in microgravity and may contribute to decreased de-gassing efficiency. It was shown from this study that the radial membrane contactor does degas the FC72 during microgravity conditions even though it is not as efficient as during normal gravity conditions.

\section{References}

[3M, 2013] 3M. Flourinert FC-72. (Material Safety Data Sheet). Retrieved from

http://amsmaterials.com/products/Fluorinert-FC\%252d72.html.

[Liqui-cel, 2013] Liqui-cel Membrana, Charlotte, South Carolina.

[National Instruments, 2013] National Instruments, Inc., Austin, TX.

[TSLOTS, 2013] TSlots/Futura Industries, Clearfield, Utah. 